\title{
Physics potential of ATLAS upgrades at HL-LHC
}

\author{
M.Testa; \\ INFN LNF, Italy \\ E-mail: marianna.testa@lnf.infn.it
}

The High Luminosity-Large Hadron Collider (HL-LHC) is expected to start in 2026 and to provide an integrated luminosity of $3000 \mathrm{fb}^{-1}$ in ten years, a factor 10 more than what will be collected by 2023. This high statistics will allow ATLAS to perform precise measurements in the Higgs sector and improve searches for new physics at the TeV scale. The luminosity needed is $\mathrm{L} \sim 7.510^{34} \mathrm{~cm}^{-2} \mathrm{~s}^{-1}$, corresponding to $\sim 200$ additional proton-proton pile- up interactions. To face such harsh environment some sub-detectors of the ATLAS experiment will be upgraded or completely substituted. The performances of the new or upgraded ATLAS sub-detectors are presented, focusing in particular on the new inner tracker and a proposed high granularity time device. The impact of those upgrades on crucial physics measurements for HL-LHC program is also shown.

The European Physical Society Conference on High Energy Physics

5-12 July, 2017

Venice

*on behalf of the ATLAS Collaboration 


\section{Introduction}

The HL-LHC will deliver an integrated luminosity of $3000 \mathrm{fb}^{-1}$ in ten years starting from 2026, to perform precise measurements in the Higgs sector and improve searches for new physics at the TeV scale. About 200 pile-up collisions are expected at the HL-LHC, to be compared with $\sim 40$ during Run 2. The ATLAS Phase-2 Scoping Document [1] provides an overview of the plans for this high luminosity phase. The current Inner Detector will be replaced by a new all-silicon tracker (ITk) to maintain tracking performance in this high-occupancy environment and to cope with the increase of approximately a factor of ten in the integrated radiation dose. The ITk will have a larger angular coverage, up to $|\eta|<4$, to mitigate efficiently pile-up effects, especially in the jet and Missing Transverse Energy (MET) reconstruction and to provide a better reconstruction of forward leptons. The ITk will have a better impact parameter resolution with respect to current Inner Tracker due to reduced material budget. In particular the improvement in the resolution of $z_{0}$ impact parameter of tracks, shown in Figure 1, is crucial to assign correctly tracks to the primary vertex. The deterioration at large $|\eta|$ is due to increased material crossed.

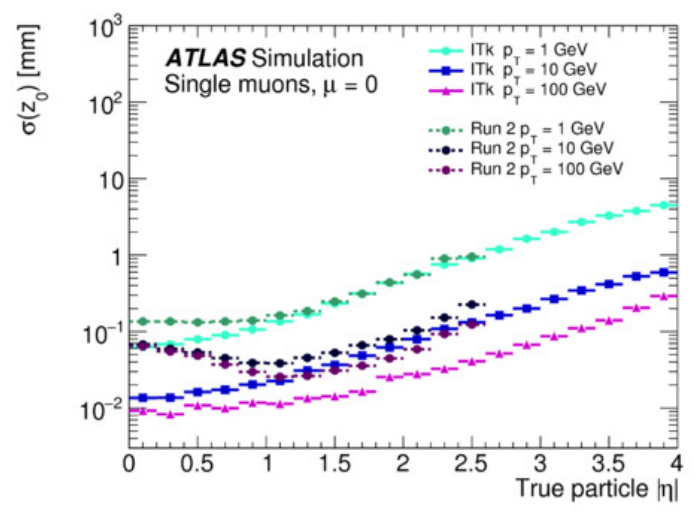

Figure 1: Resolution on the $z_{0}$ impact parameter as a function of $|\eta|$ for muons for various transverse momentum, for ITk and current Run2 inner tracker [2].

\section{ITk Performance on pile-up jet suppressions}

Tracks, and their vertex association information, can be used to mitigate pile-up effects in the jet and MET reconstruction. During Runs 1 and 2, ATLAS developed several jet vertex-tagging methods to identify and remove pile-up jets $[3,4]$. A major limitation of the current track-based pile-up suppression methods for jets is that they are not applicable to jets outside the inner tracker detector acceptance. The extension from the current $|\eta|<2.5$ tracker acceptances to $|\eta|<4$ of the ITk will enable these techniques to be employed to reject pile-up jets in the forward region as well. An example of jet vertex-tagger is given by the discriminant defined as the scalar sum of the $p_{T}$ values of all tracks that are inside the jet cone and originate from the hard-scatter vertex, divided by the calibrated jet $p_{\mathrm{T}}$. This discriminant will be referred as $R_{\mathrm{pT}}$ in the following. The tracks are required to have a transverse momentum larger than $1 \mathrm{GeV}$. The distance between the hard-scatter 
vertex and the $z_{0}$ impact parameter of the tracks used in the $R_{\mathrm{pT}}$ calculation is required to be within $1 \mathrm{~mm}$ and $4 \mathrm{~mm}$, depending on the $|\eta|$ of the track. Details are given in [2].

The efficiency for pile-up jets as a function of the efficiency for hard-scatter jets with $20<$ $p_{\mathrm{T}}<40 \mathrm{GeV}$, in three different $|\eta|$ regions, using the $R_{\mathrm{pT}}$ discriminant is shown in Figure 2, left. It is seen that even at high $\eta$, beyond the present tracker acceptance, a good discrimination is achieved. MET reconstruction is very sensitive to pile-up jets multiplicity. The resolution and tails of MET can be reduced by improving pile-up jet suppressions. In this analysis MET is reconstructed as in [2]. Figure 2, right, shows that the resolution is about $30 \%$ better when using tracks up to $\eta=4$.0. This improvement is primarily explained by the removal of forward pile-up jets.
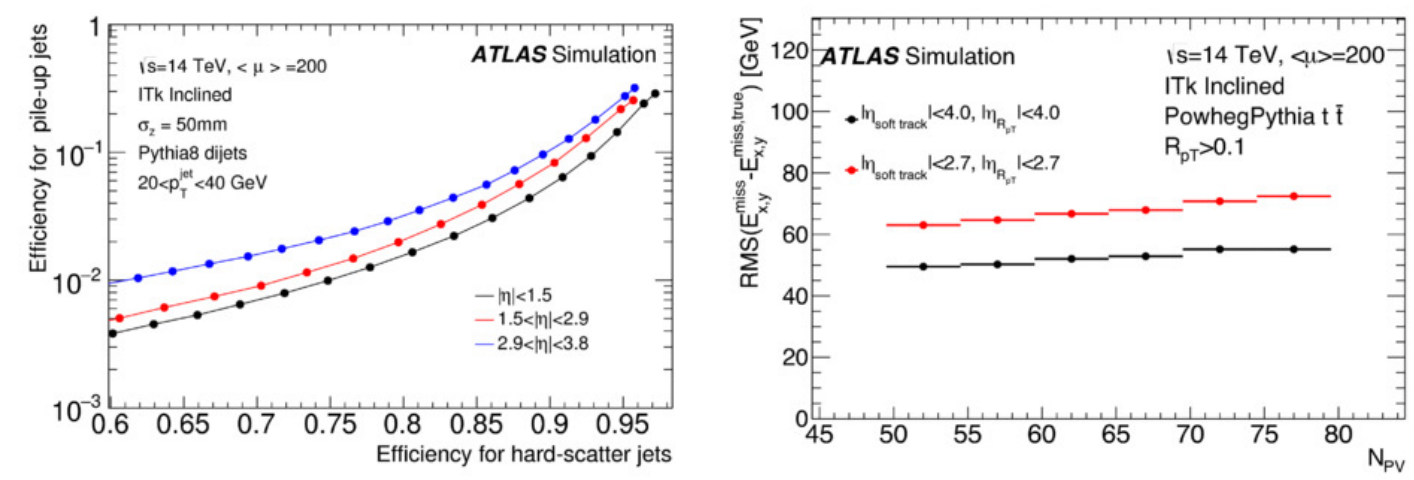

Figure 2: Left: The efficiency for pile-up jets as a function of the efficiency for hard-scatter jets with $20<p_{T}<40 \mathrm{GeV}$ [2]. Right: The resolutions of the $\mathrm{x}$ and $\mathrm{y}$ components of MET for the $|\eta|<2.7$ (red) and $|\eta|<4.0$ (black) Inner Tracker acceptance ranges for samples of Powheg+Pythia $t \bar{t}$ events with $\langle\mu\rangle=200$, as a function of the number of reconstructed primary vertices [2].

\section{HGTD Performance on pile-up jet suppressions}

A proposed upgrade for HL-LHC is the High Granulatiry Time Device (HGTD), with thickness $6 \mathrm{~cm}$, located in front of endcap calorimeter at $2.4<|\eta|<4$. The HGTD is equipped with Low Gain Avalanche Diodes Cell with size $1 \times 1 \mathrm{~mm}^{2}$, providing a timing resolution of $\sim 30 \mathrm{ps}$. A first design was given in [1]. The HGTD initial design report is being finalizing. The motivation for this upgrade is to use timing information to improve the pile-up suppression in the offline and online reconstruction. At $\mu=200,5-7$ vertices within tracker resolution at large $|\eta|$ are expected to be merged, due to larger $z_{0}$ resolution. Degraded performance of pile-up suppression techniques and isolation efficiency at large $|\eta|$ are therefore expected. Timing information may help pile-up rejection resolving nearby vertices. Timing information could also improve trigger capabilities at 40 $\mathrm{MHz}$, where the tracking information is not yet available. The impact of HGTD to improve pile-up jet rejection have been addressed using the $R_{\mathrm{pT}}$ discriminant defined above, using in combination the timing information of the tracks . Figure 3 shows the efficiency for hard-scatter jets versus $\eta$ for jets with $20<p_{T}<50 \mathrm{GeV}$ for a $2 \%$ pile-up jets rejection efficiency. The vertices are normally distributed along the beam axis and in time with $\sigma_{z}=50 \mathrm{~mm}, \sigma_{t}=180$ ps with $\mu=200$. The black curve is obtained using tracking information only. The tracks used in the $R_{\mathrm{pT}}$ calculation fulfill 
the same requirements reported in Section 2. For the red curve, reconstructed tracks are selected as for the black curve and with a timing consistent within $60 \mathrm{ps}$ with the hard-scatter vertex truth time. The time resolution of the tracks is assumed to be $30 \mathrm{ps.} \mathrm{An} \mathrm{improvement} \mathrm{using} \mathrm{HGTD} \mathrm{in}$ the efficiency to select hard-scatter jets is observed at high $|\eta|$. In this region, due to the $z_{0}$ impact resolution degradation, the impact of timing information to correctly assign the track to the primary vertex is larger.

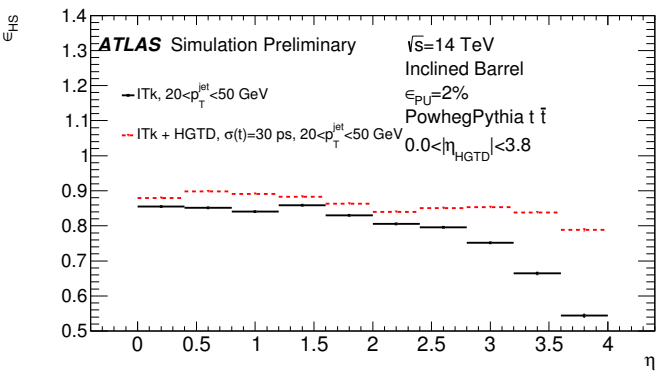

Figure 3: The efficiency to select hard-scatter jets with $20<p_{T}<40 \mathrm{GeV}$, as a function of $|\eta|$ for a $2 \%$ pile-up jets rejection efficiency with and without HGTD [5]. See text for details.

\section{Physics channels}

The impact of the ITk to physics channels, crucial for the HL-LHC program, has been studied in [2]. Particular focus has been given to the impact of the extended ITk acceptance to $|\eta|=4$. For this propose, the Vector Boson Scattering (VBS) process and Vector Boson Fusion (VBF) process for the Higgs boson production, with forward jets in the final states, have been studied in details.

VBS is a crucial process to understand the nature of the electroweak symmetry breaking mechanism.The $W^{ \pm} W^{ \pm}$-electroweak scattering process with two same charge leptons in the final states has been considered. The main background comes from $W Z+$ jets production. The expected uncertainty on the cross section with and without the forward tracking, is $4.5 \%$ and $3.9 \%$ respectively. The improvements come from the possibility to reject efficiently pile-up jets in the forward region and to veto additional leptons in the forward region.

The accurate measurement of the Higgs production via VBF is crucial for the HL-LHC program. The expected precision in the cross section measurement with tracking coverage up to $|\eta|<4.0,3.2$ and 2.7 is respectively 12,18 and $22 \%$. No theoretical uncertainty is considered. The improvements come from a more efficient central jet veto, due to the better forward pile-up jet rejection, and from the possibility to apply a b-jet veto also in the forward region which gives better $t \bar{t}$ background suppression.

\section{References}

[1] ATLAS Collaboration, “ATLAS Phase-II Upgrade Scoping Document”, CERN-LHCC-2015-020, https://cds.cern.ch/record/2055248 
[2] ATLAS Collaboration, "Technical Design Report for the ATLAS Inner Tracker Strip Detector", CERN-LHCC-2017-005, https://cds.cern.ch/record/2257755

[3] ATLAS Collaboration, "Tagging and suppression of pileup jets with the ATLAS detector", ATLAS-CONF-2014-018, https://cds.cern.ch/record/1700870

[4] ATLAS Collaboration, "Forward Jet Vertex Tagging: A new technique for the identification and rejection of forward pileup jets”, ATL-PHYS-PUB-2015-034, https://cds.cern.ch/record/2042098

[5] ATLAS Collaboration, "LArHGTDPublicPlots", https://twiki.cern.ch/twiki/bin/view/AtlasPublic/LArHGTDPublicPlots 